\title{
Viscoelastically generated prestress from ultra-high molecular weight polyethylene fibres
}

\author{
Adnan Fazal $\cdot$ Kevin S. Fancey
}

Received: 18 January 2013/Accepted: 25 March 2013/Published online: 6 April 2013

(c) Springer Science+Business Media New York 2013

\begin{abstract}
The viscoelastic characteristics of ultra-high molecular weight polyethylene (UHMWPE) fibres are investigated, in terms of creep-induced recovery strain and force output, to evaluate their potential for producing a novel form of prestressed composite. Composite production involves subjecting fibres to tensile creep, the applied load being removed before moulding the fibres into a resin matrix. After matrix curing, the viscoelastically strained fibres impart compressive stresses to the surrounding matrix, to produce a viscoelastically prestressed polymeric matrix composite (VPPMC). Previous research has demonstrated that nylon fibre-based VPPMCs can improve mechanical properties without needing to increase mass or section dimensions. The viability of UHMWPE fibre-based VPPMCs is demonstrated through flexural stiffness tests. Compared with control (unstressed) counterparts, these VPPMCs typically show increases of $20-40 \%$ in flexural modulus. Studies on the viscoelastic characteristics indicate that these fibres can release mechanical energy over a long-timescale and fibre core-skin interactions may have an important role.
\end{abstract}

\section{Introduction}

Viscoelastically prestressed polymeric matrix composites (VPPMCs) offer the means to improve the mechanical performance of composite structures without the need to increase mass or section dimensions. VPPMC production involves subjecting polymeric fibres to tensile (viscoelastic) creep; the creep load is then released prior to the fibre-

A. Fazal · K. S. Fancey $(\square)$

School of Engineering, University of Hull, Hull HU6 7RX, UK

e-mail: k.s.fancey@hull.ac.uk matrix moulding process. Following matrix curing, compressive stresses are imparted to the surrounding matrix as the strained fibres (in residual tension) attempt viscoelastic recovery.

This state of matrix compression-fibre tension can also be achieved with elastically prestressed PMCs (EPPMCs): here, the required prestress is obtained by stretching fibres within the composite to maintain an elastic strain as the matrix cures. Studies with unidirectional glass fibre EPPMCs, compared with unstressed counterparts, have indicated tensile strength and elastic modulus increases of $\sim 25$ and $\sim 50 \%$, respectively [1]. Similarly, increases of up to $33 \%$ have been found for impact resistance and flexural properties (stiffness and strength) [2,3]. These improvements have been explained through the effects of matrix compression and fibre tension on (i) impeding or deflecting propagating cracks and (ii) reducing composite strain caused by external tensile or bending loads [1-3].

Mechanisms to explain the improvements obtained from VPPMCs also follow similar reasoning. Compared with EPPMCs, however, VPPMCs offer two potentially significant benefits [4]. First, since the VPPMC fibre stretching and moulding operations are totally separate, there are no constraints on fibre length, orientation, and spatial distribution during the moulding process that could restrict composite geometry. Second, a gradual deterioration in prestress would be expected within an EPPMC, due to matrix creep in the vicinity of the fibre-matrix interface. A VPPMC would be much more resistant, due to matrix creep effects being counterbalanced by longer term viscoelastic recovery mechanisms in the polymeric fibres.

The initial evaluation method used in VPPMC research was Charpy impact testing. These tests demonstrated that VPPMC samples could absorb typically 25-30\% more impact energy than their control (unstressed) counterparts, 
with some samples reaching increases of $50 \%$ [4-7]. Subsequent tensile testing [8] showed increases in strength, modulus and energy absorbed (to 0.25 strain) to exceed 15 , 30 and $40 \%$, respectively, and flexural modulii from threepoint bend tests [9] were found to be $\sim 50 \%$ greater than corresponding control samples. More recent studies have returned to Charpy impact testing, to investigate (i) longterm behaviour and (ii) impact energy absorption mechanisms. For (i), accelerated ageing (using time-temperature superposition principles) has demonstrated no deterioration in VPPMC impact performance over a duration equivalent to $40{ }^{\circ} \mathrm{C}$ ambient for $\sim 20$ years [10]. The main finding for (ii) has been that improved energy absorption depends principally on shear-stress induced fibre-matrix debonding [11].

As of date, research into VPPMCs has been restricted to investigations with prestress provided by nylon 6,6 fibres. This paper reports the first findings from investigations with ultra-high molecular weight polyethylene (UHMWPE) fibres.

\section{Background}

UHMWPE fibre treatment and analysis of viscoelastic characteristics

Viscoelastically generated prestress requires fibres to store mechanical energy so that it can be released over a very long timescale. Thus, after removing a tensile creep load and undergoing instantaneous (elastic) recovery, potentially suitable fibres should exhibit a significant proportion of long-term viscoelastic recovery strain followed by zero (or almost zero) steady-state strain from viscous flow effects. Previous investigations with nylon 6,6 showed that as-received fibres exhibited notably lower creep and recovery strain values compared with fibres that had been annealed prior to identical loading conditions; also, recovery for the as-received fibres approached strain levels close to zero within $1000 \mathrm{~h}$ of releasing the creep load [4, $6,7,10]$. In contrast, recovery strain (hence viscoelastic activity) from annealed fibres is shown to remain active beyond 1000 years at $20^{\circ} \mathrm{C}$ [10].

Clearly, the treatment of UHMWPE fibres must be given similar consideration. For nylon 6,6 fibre VPPMC studies, annealing conditions $\left(150{ }^{\circ} \mathrm{C}\right.$ for $\left.0.5 \mathrm{~h}\right)$ were deduced from sources cited therein [4-10]. For UHMWPE fibres, there is less certainty. Gupta [12] suggests that any meaningful heat-setting (to remove structural instabilities) of highdensity polyethylene fibres would be performed at $\sim 120{ }^{\circ} \mathrm{C}$; however, it may be inferred from Ref. [12] that the need to anneal UHMWPE fibres is more questionable, due to their high crystallinity. Thermal treatment $(0.25 \mathrm{~h})$ of UHMWPE fibres shows that tensile strength is unaffected, though modulus decreases and strain-to-break increases progressively with increasing temperature up to $130{ }^{\circ} \mathrm{C}$ [13]. Annealing at $100{ }^{\circ} \mathrm{C}$ is found to relax some of the strain in the intermediate (oriented amorphous) phase between crystals, which results in a brittle to plastic transition within these regions [14]. By considering these aspects, the annealing conditions for this work were set to $120{ }^{\circ} \mathrm{C}$ for $0.5 \mathrm{~h}$.

The first practical requirement was to establish suitable load-time conditions for long-term viscoelastic energy storage. This is most easily achieved through strain-time measurements during recovery from an applied creep load. The resulting recovery strain data, $\varepsilon_{\text {rvis }}(t)$, as a function of time, $t$, may then be fitted to:

$\varepsilon_{\text {rvis }}(t)=\varepsilon_{\mathrm{r}}\left[\exp \left(-\left(\frac{t}{\eta_{r}}\right)^{\beta_{\mathrm{r}}}\right)\right]+\varepsilon_{\mathrm{f}}$

Equation (1) comes from the Weibull or KohlrauschWilliams-Watts (KWW) relationship, where polymeric deformation can be represented by a model consisting of time-dependent mechanical latch elements $[15,16]$. Viscoelastic strain recovery is represented by the $\varepsilon_{\mathrm{r}}$ function, which depends on the Weibull shape parameter, $\beta_{\mathrm{r}}$, and characteristic life, $\eta_{\mathrm{r}}$. The permanent strain from viscous flow effects, $\varepsilon_{\mathrm{f}}$, is the residual strain as $t$ approaches $\infty$ and is ideally zero. Thus, Eq. (1) enables $\varepsilon_{\mathrm{f}}$ to be predicted from shorter-term recovery strain data.

After establishing the most appropriate loading conditions, the viscoelastic recovery force from UHMWPE fibres was investigated using a bespoke force measurement (FM) rig $[17,18]$. Following creep and elastic recovery, the remaining time-dependent recovery force was monitored at a fixed strain, by means of a force sensor. The required creep-recovery test cycle is represented by Fig. 1. Previous work with nylon 6,6 showed that the force grew to $3.4 \%$ of applied creep load over a $2700 \mathrm{~h}$ measurement period $t$, and was predicted to approach a maximum of $3.8 \%$ as $t \rightarrow \infty$ [18]. This prediction was based on fitting recovery force data in Ref. [18] to the following:

$\sigma(t)=\sigma_{\mathrm{v}}\left[\exp \left(-\left(\frac{\Delta t}{\eta}\right)^{\beta}\right)-\exp \left(-\left(\frac{t}{\eta}\right)^{\beta}\right)\right]$

Equation (2) shares the same origins as Eq. (1). Here, $\sigma(t)$ represents the time dependent recovery stress (force across the fibre cross-sectional area) from the $\sigma_{\mathrm{v}}$ function, as determined by the characteristic life $(\eta)$ and shape $(\beta)$ parameters. The time delay between releasing the creep load and establishing the onset of recovery force is represented by $\Delta t$ in Eq. (2) and Fig. 1. 


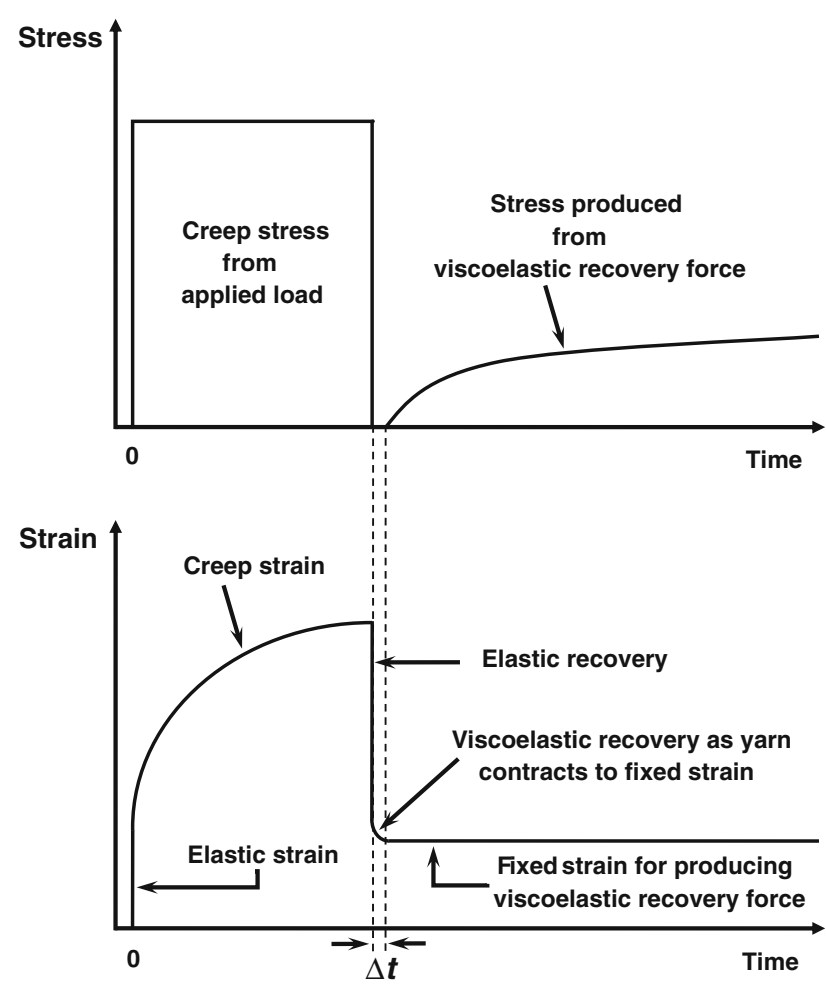

Fig. 1 The creep-recovery test cycle to investigate the force-time characteristics of viscoelastically recovering UHMWPE yarn

\section{Composite sample production and evaluation}

Open casting offered the simplest composite sample production method. The resulting beam-shaped samples enabled the same mechanical evaluation procedures to be used in previous studies with nylon 6,6 fibre composites [4-7, 9-11] so that comparative assessments could be made. As with previous studies, mechanical evaluation required comparing the performance of VPPMC 'test' samples with unstressed 'control' counterparts. Clearly, this assumes no differences between test and control samples, other than the effects of prestress in the former case. To verify this necessitated (i) microscopic inspection of fibres and moulded cross-sections for any changes due to the stretching process and (ii) tensile testing of fibres to ensure that the stretching process did not, for example, cause work-hardening.

Although Charpy impact testing was the principal VPPMC mechanical evaluation method for nylon fibrebased VPPMCs [4-7, 10, 11], flexural stiffness measurements have been adopted instead for this investigation. The latter was preferred here, since samples would not be destroyed during testing and thus could be repeatedly measured to correlate possible time-dependent changes with viscoelastic recovery data. In contrast, a substantial programme of work would be required to provide opportunities for elucidating the mechanisms associated with observations from impact testing.

Previous flexural stiffness measurements for nylon fibre VPPMCs involved three-point bend tests on samples using a freely suspended load. To determine (as close as possible) the elasticity modulus, a deflection reading was taken $5 \mathrm{~s}$ after applying the load [9] and the same principle was adopted for this work. From the conventional three-point beam-bending relationship [19], the flexural modulus $E(t)$ can be determined from deflection $\delta(t)$ at the centre of the beam at time $t$ (i.e. 5 s) using:

$$
E(t)=\frac{P L^{3}}{48 \delta(t) I}
$$

where $P$ is the applied load, $L$ is the span, and $I$ is the second moment of area, which is $\left(b h^{3} / 12\right)$ for a rectangular sample of width $b$ and thickness $h$.

\section{Experimental}

Assessment of creep and recovery strain

Fibre used for this study was a continuous multi-filament UHMWPE untwisted yarn, i.e., Dyneema SK60 supplied by Goodfellow Cambridge Ltd., UK. The yarn had 1600 filaments (fibres) with $12 \mu \mathrm{m}$ mean filament diameter (supplier specification). Creep-recovery procedures were similar to those previously used for equivalent nylon 6,6 fibre studies [4-7]. For annealing, a suitable length of yarn was placed, unconstrained, in an aluminium tray and maintained at $120{ }^{\circ} \mathrm{C}$ for $0.5 \mathrm{~h}$ in a fan-assisted oven. The yarn was then attached to a loading rig with counterbalanced platform to accommodate weights for creep (designated Rig $A$ ). Creep and recovery strain measurements could be made in situ by measuring the distance between two inked marks on the yarn, typically $300-400 \mathrm{~mm}$ apart, with a digital cursor $( \pm 0.01 \mathrm{~mm}$ precision). All strain measurements were made under ambient conditions of $19.5-21.0^{\circ} \mathrm{C}$ and $35-55 \% \mathrm{RH}$.

Three separate samples of yarn were successively annealed and loaded under identical creep conditions of $1.36 \mathrm{GPa}$ for $24 \mathrm{~h}$. Creep strain measurements were made and, on releasing the load, measurements of recovery strain were subsequently taken. The high strain rates encountered during initial stages of measurement allowed only individual readings to be recorded for strain values during the first hour of recovery (and creep). Strain rates after $1 \mathrm{~h}$ were considered to be sufficiently low to enable each strain value to be determined from the mean of three readings. To evaluate the effects of annealing, three further samples of yarn were subjected to the same creep-recovery procedures, with the annealing stage omitted. 
As with earlier modelling studies $[4,7,15,16]$, Eq. (1) was fitted to the recovery strain data using commercially available software (CurveExpert 1.4). In addition to providing equation parameter information, the resulting correlation coefficient indicated the quality of fit between equation-predicted and measured strain-time values.

\section{Production of composite samples}

Batches of composite samples were required for flexural stiffness evaluation and two fibre volume fraction $\left(V_{\mathrm{f}}\right)$ values were used. The higher value $(7.2 \%)$, was comparable to those used in previous nylon fibre VPPMC studies involving flexural stiffness [9], whilst the lower (3.6\%), was similar to that used in recent Charpy impact investigations [11].

The same annealing procedures were followed as for creep and recovery assessment, though two lengths of yarn (test and control) were simultaneously annealed. Since larger quantities of yarn were required, a bespoke stretching rig (using a system of pulleys) was used (designated Rig $B$ ); this was previously employed in the production of higher $V_{\mathrm{f}}$ nylon fibre VPPMCs $[8,9,11]$. The test yarn was subjected to a $24 \mathrm{~h}$ creep load of $1.3 \mathrm{GPa}$, whilst the control yarn was positioned close to the rig for exposure to the same ambient conditions. Both yarns were then folded, cut to appropriate lengths and combed into flat ribbons for moulding.

A clear-casting polyester resin was used as previously [11] for the matrix, i.e., Cray Valley Norsodyne E9252, mixed with $1 \%$ MEKP catalyst, supplied by CFS Fibreglass Supplies, UK. Gel time (at room temperature) was $\sim 0.25 \mathrm{~h}$ and after $2 \mathrm{~h}$, the resin was sufficiently cured to permit demoulding. Two aluminium moulds were used for the open casting of unidirectional continuous fibre composite samples. Each mould had a $10 \mathrm{~mm}$ wide channel for casting a strip of test and control materials simultaneously from the same resin mix. Casting was completed within $0.5 \mathrm{~h}$ of the fibre stretching process and, after demoulding, the strips were cut into appropriate lengths and held under a weighted steel strip for $24 \mathrm{~h}$ to prevent any residual stresses causing sample distortion.

At $7.2 \% V_{\mathrm{f}}$, the high loads required for stretching UHMWPE fibre limited production to just one test and one control sample per batch, each sample being $200 \times 10 \times$ $3.2 \mathrm{~mm}$. Although this limitation did not apply to $3.6 \% V_{\mathrm{f}}$, the same methodology was adopted, to be consistent with production procedures.

\section{Photographic studies}

Photographic evidence of effects that could adversely influence composite sample characteristics was required, using scanning electron microscopy (SEM) and optical microscopy $(\mathrm{OM})$. This was to ensure that there would be no differences between test and control samples, other than mechanical effects from prestress. SEM was used to assess potential changes in topography of test yarn filaments following the applied creep stress. Ground and polished composite sample cross-sections were taken from the moulded strips to observe, by OM, fibre spatial distributions.

\section{Mechanical evaluation of fibres}

Nylon 6,6 studies involved tensile testing of individual test and control fibres to ensure no changes in the former from effects such as work-hardening [8]. This was not possible with UHMWPE fibres, due to dimensional (cross-sectional) variations between individual filaments. These would cause difficulties in determining cross-sectional area; also test and control filament cross-sectional geometries would (ideally) need to be matched to enable direct comparison. Thus macroscopic tensile testing of test and control yarns had to be performed, the test yarn being produced from Rig $B$.

Compared with most materials, yarns are more sensitive to stress concentrations when clamped and stretched during tensile testing, though the capstan method can be an effective technique [20]. This principle was adopted as shown in Fig. 2a, the capstan design and dimensions being comparable to those used elsewhere for UHMWPE fibre evaluation [21]. Although tensile strength $\left(\sigma_{\mathrm{f}}\right)$ would be unaffected, a potential problem with this arrangement was the uncertainty in gauge length, which was required for determining the Young's modulus $(E)$ and strain-to-failure $\left(\varepsilon_{\mathrm{f}}\right)$. During tensile testing, fibre movement around the capstans makes the effective gauge length $\left(L_{0 \mathrm{e}}\right)$ greater than the apparent gauge length $\left(L_{0 \text { ap }}\right)$ shown in Fig. 2a. For evaluation of single UHMWPE filaments in Ref. [21], however, $L_{0 \mathrm{e}}$ was found to be equivalent to the total length, i.e. $L_{0 \text { ap }}$ plus length of material wound around the capstans. For the purposes of our work, in which the principal aim was to determine possible differences between yarns, the assumption that $L_{0 \mathrm{e}}$ is equal to the total length was adopted. Individual lengths of yarn (4 test and 4 control) were tested in succession using the capstan jigging in a Lloyd LR100 K machine (with analysis software) at $20-21{ }^{\circ} \mathrm{C}$. The total length for each yarn sample was $650 \mathrm{~mm}$ $\left(L_{0 \mathrm{ap}}=130 \mathrm{~mm}\right)$ and the loading rate was $200 \mathrm{~mm} / \mathrm{min}$. The testing was performed $168 \mathrm{~h}$ (1 week) following stretching procedures and the resulting stress-strain curves provided information on $\sigma_{\mathrm{f}}, E$, and $\varepsilon_{\mathrm{f}}$.

Recovery force from fibres

Following annealing, the yarn was (i) subjected to a $24 \mathrm{~h}$ creep load, (ii) the load was then released (to allow elastic recovery) and (iii) the viscoelastically recovering material was transferred to the FM rig for force-time monitoring. 
(a)

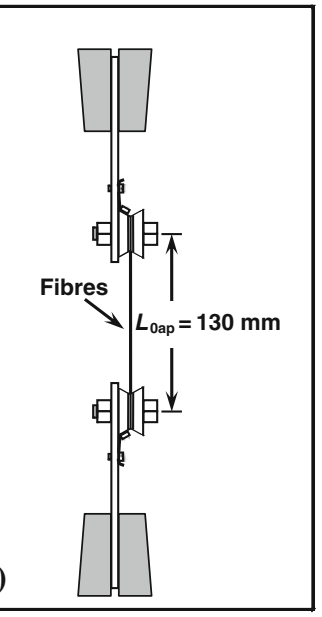

(b)
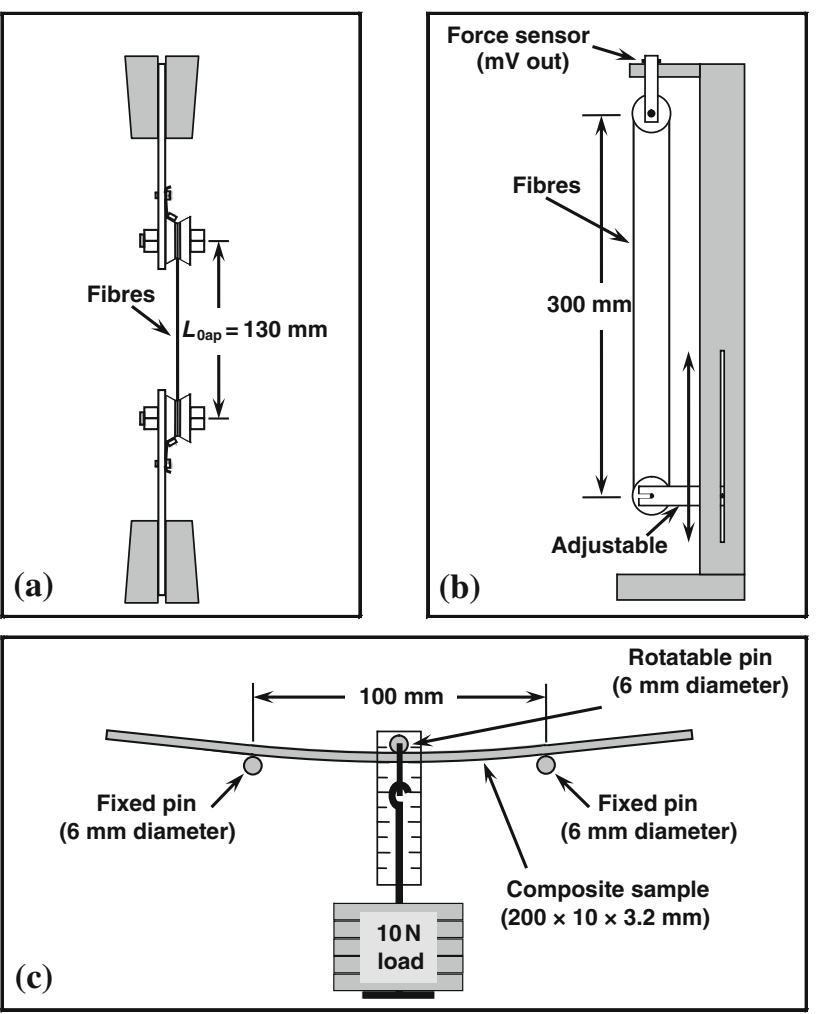

Fig. 2 Schematics of test equipment used in this study a jig assembly for tensile testing of yarn samples, $\mathbf{b}$ force measurement (FM) rig used for measuring viscoelastic recovery force, $\mathbf{c}$ three-point bend testing of composite samples

Rig $A$ was utilised for (i) as this was compatible with the FM rig bobbin fixtures that enabled direct transfer of the recovering yarn in (iii). Full FM rig details are given in Ref. [18], though essentially, the rig consisted of a loop of yarn, supported by upper and lower bobbins, attached to a force sensor (Fig. 2b).

Owing to the high loading required for stretching UHMWPE fibre, combined with weight limitations for Rig $A$, the yarn had to be separated out (before annealing) to reduce cross-sectional area by $\sim 50 \%$. This was then attached to the FM upper and lower bobbins as a single loop (thus providing the approximate cross-sectional area of one yarn) and fitted to Rig $A$ for a $24 \mathrm{~h}$ creep loading of $1.36 \mathrm{GPa}$. On releasing the creep load, the bobbin assembly was transferred to the FM rig. During time $\Delta t$ (Fig. 1), the initially loose loop of yarn progressively tightened through viscoelastic recovery, until a force output could begin to be monitored at a fixed strain; all readings were recorded at $20.9 \pm 1.0^{\circ} \mathrm{C}$. Subsequently, Eq. (2) could be fitted to the resulting data, with the same software used for Eq. (1).

Mechanical evaluation of composites

Three-point bend tests were performed using a simple test rig with a freely suspended load (Fig. 2c). The set-up and procedures were identical to those performed with nylon fibre VPPMC (long length) samples [9], i.e., each sample was mounted horizontally with the moulded bottom surface facing downwards and a deflection reading was taken at $5 \mathrm{~s}$ after applying the load. Although small deflections restricted measurement precision and accuracy, a low load was used in Ref. [9] ( $\sim \mathrm{N})$ to minimise opportunities for specimen damage. To achieve comparable deflections from samples in the present study, a load of $10 \mathrm{~N}$ was adopted.

Deflections were measured at $20-21^{\circ} \mathrm{C}$ on each sample just once at $336 \mathrm{~h}, 1008$ and $2016 \mathrm{~h}$ and a video recording of the deflection in progress was made, to improve measurement accuracy. For repeatability, three test and three control samples (i.e., three batches) were evaluated at both $V_{\mathrm{f}}$ values.

\section{Results}

Creep and recovery strain

The creep and recovery strain-time results are shown in Figs. 3 and 4. Scatter in the data points can be attributed to uncertainty in locating ink mark edges on these multifilament yarns during strain measurement. This scatter was exacerbated during strain recovery (Fig. 4), since data were also sensitive to ensuring that the yarn was maintained in a straight position during strain measurement. Comparing with results using nylon 6,6 yarns $[4,6,7,10]$, there is greater data dispersion in recovery. This arises from the UHMWPE yarn characteristics, i.e., a high number of very fine filaments leading to their greater susceptibility to becoming separated from repeated handling. Separation is exacerbated, since handling increases the presence of kink bands along the filaments, an effect also observed by others [22].

Despite the scatter in Fig. 4, the most important observation is that the yarn undergoes time-dependent strain recovery, the strain-time magnitudes being greater with annealed yarn. The applied creep stress $(1.36 \mathrm{GPa})$ was almost four times the value used in nylon 6,6 studies (342 MPa), though the $24 \mathrm{~h}$ creep strain for annealed UHMWPE in Fig. 3, at $5.4 \%$, is substantially lower than the $12.4 \%$ observed for annealed nylon 6,6 [4, 6]. Nevertheless, recovery strain-time levels are comparable; e.g., at 0.1 and $1000 \mathrm{~h}$, respectively, UHMWPE gives 2.3 and $1.7 \%$ in Fig. 4, compared with 2.8 and $1.6 \%$ for nylon 6,6 $[7,10]$.

Some comparison with other UHMWPE creep studies can be made. Berger et al. [23] studied single-filament creep at $1.5 \mathrm{GPa}$ applied stress. This is close to the value used in our study $(1.36 \mathrm{GPa})$, thus creep compliance at $24 \mathrm{~h}$ from data in Ref. [23], i.e. $\sim 0.06 \mathrm{GPa}^{-1}$, enables a 


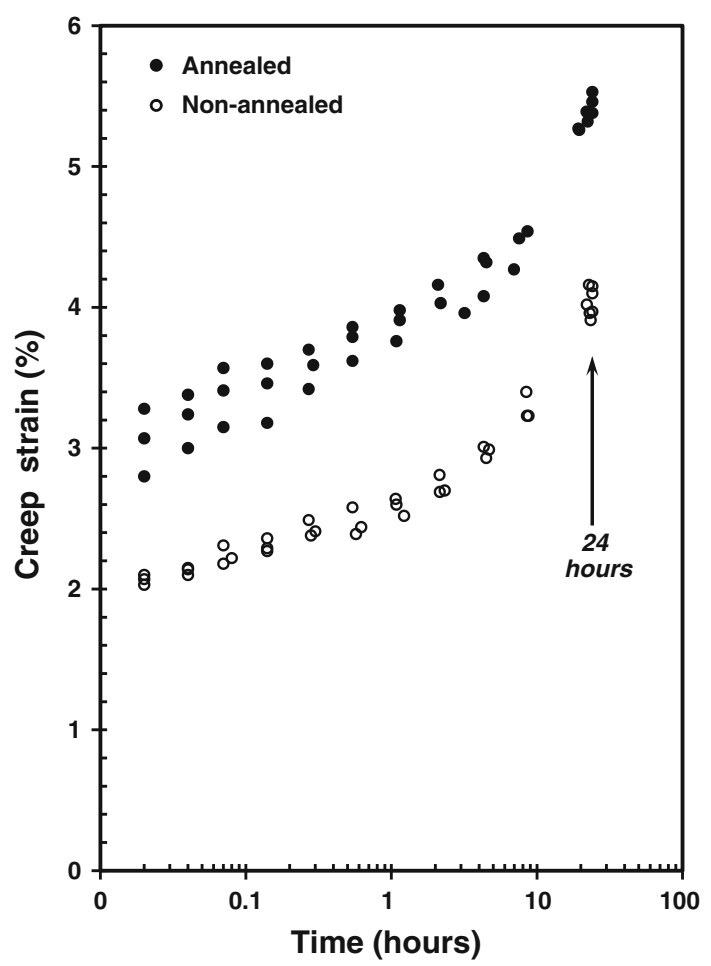

Fig. 3 Creep strain results ( $24 \mathrm{~h}$ tests) for annealed and non-annealed (as-received) UHMWPE yarn samples. The creep stress, $1.36 \mathrm{GPa}$, was determined from the applied load and unloaded cross-sectional area of the yarn

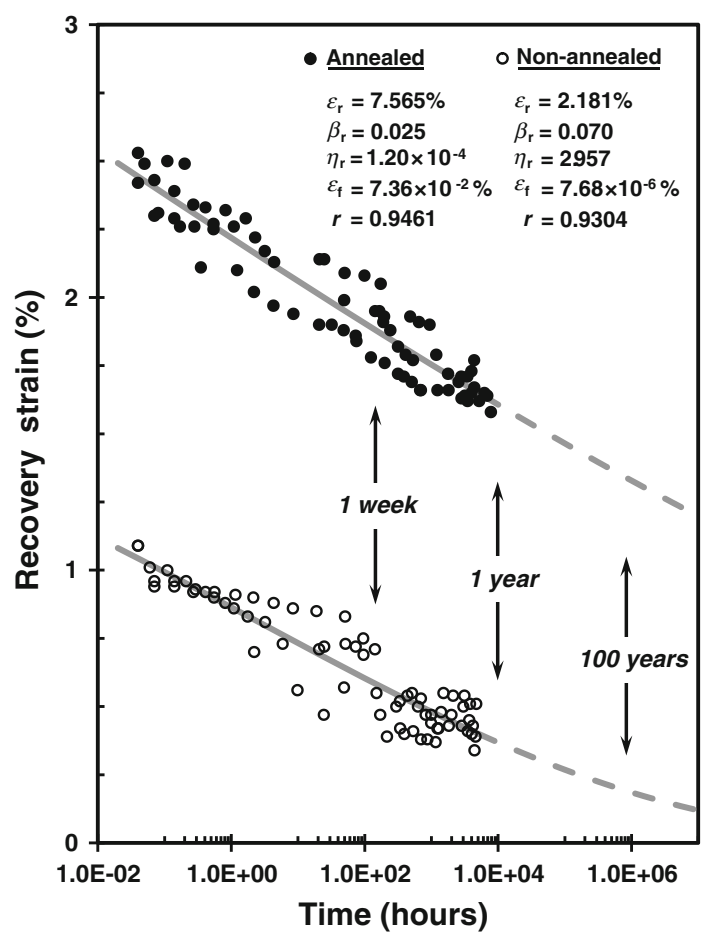

Fig. 4 Recovery strain results corresponding to the creep data of Fig. 3. The solid curves represent the Weibull model fit using Eq. (1), with listed parameters and correlation coefficient, $r$ comparison to be made with our results. From Fig. 3, the $24 \mathrm{~h}$ creep compliance for annealed and non-annealed yarns are both lower, i.e. 0.040 and $0.030 \mathrm{GPa}^{-1}$, respectively. Some discrepancy may be expected, since the applied stress value in our work was determined from a yarn cross-sectional area derived from supplier information. Pre-treatment of the material used in Ref. [23] is unspecified, but a non-annealed condition would make the equivalent compliance from our study only half their value. Our non-annealed result $\left(0.030 \mathrm{GPa}^{-1}\right)$ does, however, agree with the $24 \mathrm{~h}, 1.25 \mathrm{GPa}$ compliance value of Peijs et al. [24] for (mechanically similar) Dyneema SK66 yarn in equivalent condition.

By fitting Eq. (1) to the recovery data in Fig. 4, the indicative value for $\varepsilon_{\mathrm{f}}$ is $7.36 \times 10^{-2} \%$ (annealed) and $7.68 \times 10^{-6} \%$ (non-annealed); i.e., permanent strain from viscous flow effects is predicted to be comparatively negligible in both cases. Relevant published work is limited, though some comparison may be made with cyclic deformation studies on UHMWPE fibres [25]: here, complete viscoelastic recovery with no plastic deformation (viscous flow) was observed if the delay time between successive stress cycles ( $3.5 \mathrm{GPa})$ was $\sim 3000$ times longer than the stress cycle duration. Thus, to some extent, this lends support to our very low $\varepsilon_{\mathrm{f}}$ predictions.

\section{Comparison of test and control fibres}

Figure 5 shows SEM micrographs of (previously annealed) test and control yarn samples and there appear to be no changes to fibre topography or dimensions following the stretching treatment. Although these filaments have a supplier-specified mean diameter $(12 \mu \mathrm{m})$, they are not circular; instead, their cross-sectional geometries are bean or kidney-shaped, as described by others [23, 26].

Stress-strain plots from tensile tests performed on the yarn samples are shown in Fig. 6 and the data are summarised in Table 1. The linearity in Fig. 6 enabled $E$ to be determined up to $3 \%$ strain; this provided more consistent run-to-run results than would have been obtained from initial gradient values. Mean values obtained from the asreceived (non-annealed) samples in Table 1 are $\sim 8 \%$ lower than the supplier-specified values for $\sigma_{\mathrm{f}}(2.56 \mathrm{GPa})$ and $\varepsilon_{\mathrm{f}}(3.5 \%)$ and $\sim 13 \%$ lower for $E(87 \mathrm{GPa})$, though this may be explained by differing test conditions. The almost linear deformation response and $\varepsilon_{\mathrm{f}}$ values are similar to other non-annealed gel-spun UHMWPE fibre data [27]. In Ref. [27], fibre annealing (24 h at $\left.149{ }^{\circ} \mathrm{C}\right)$ caused $\varepsilon_{\mathrm{f}}$ to increase by $>100 \%$, whereas $\varepsilon_{\mathrm{f}}$ and other parameters in Fig. $6 \mathrm{a}$ and Table 1 shows only small differences between as-received and annealed (control) samples. Of particular interest; however, is that the data for test and control yarns are very similar in Fig. $6 \mathrm{~b}$ and Table 1. In 

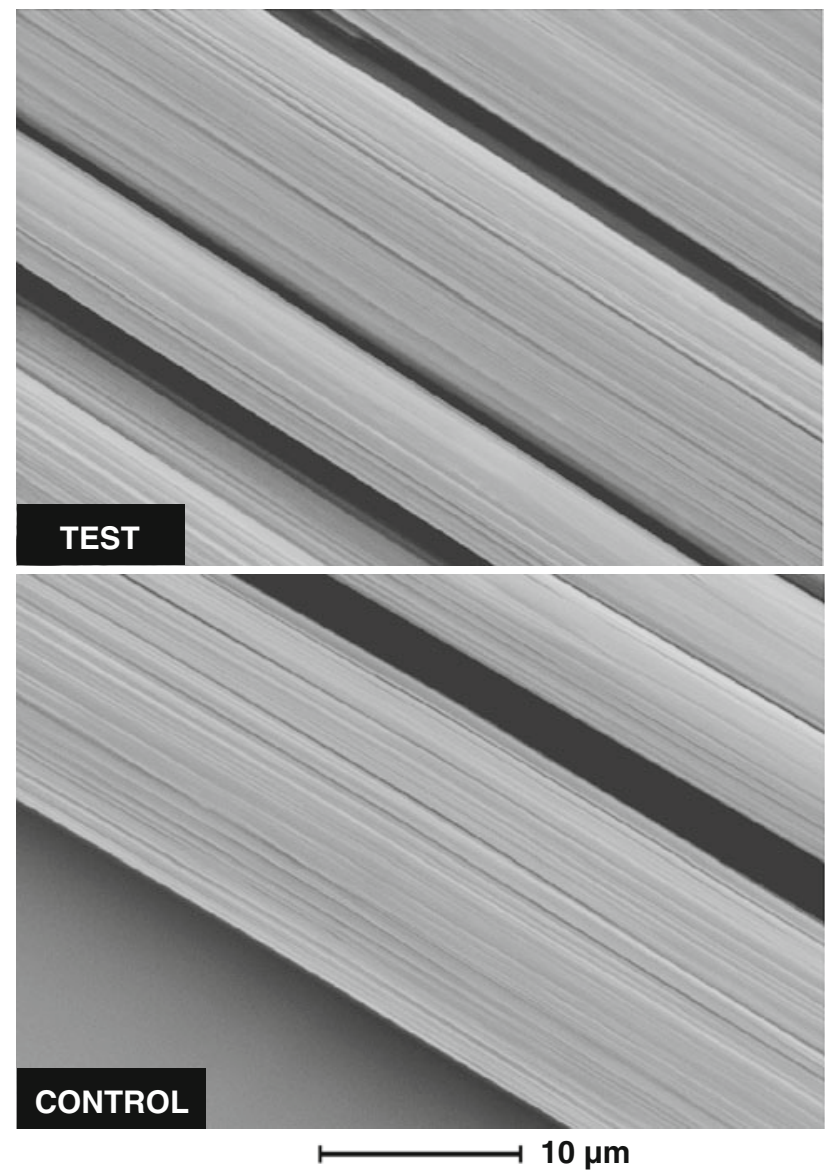

Fig. 5 SEM micrographs of test and control fibre samples, $22 \mathrm{~h}$ after releasing the creep load for the test sample

fact, although statistical analysis (hypothesis testing, $5 \%$ significance level) for the mean values of $\sigma_{\mathrm{f}}, \varepsilon_{\mathrm{f}}$, and $E$ show some differences between as-received and control yarns, there are no statistical differences between the test and control yarns for these parameters.

Typical fractured filament ends are shown in Fig. 7 and these have similar appearance to those found in the literature $[28,29]$. As illustrated by these examples, no differences were observed in the test and control fibre fracture characteristics.

\section{Recovery force}

Figure 8 shows the recovery force in terms of axial stresstime output and previous nylon 6,6 yarn results [18] are also plotted for comparison. Two observations can be made. First, the UHMWPE output is notably higher (initially) than that of the nylon and this reflects the higher creep stress ( $>4$ fold) that could be applied to the former. The second observation is that although Eq. (2) may be fitted to the first few hours of the UHMWPE plot, there is clearly a deviation from this characteristic. This could
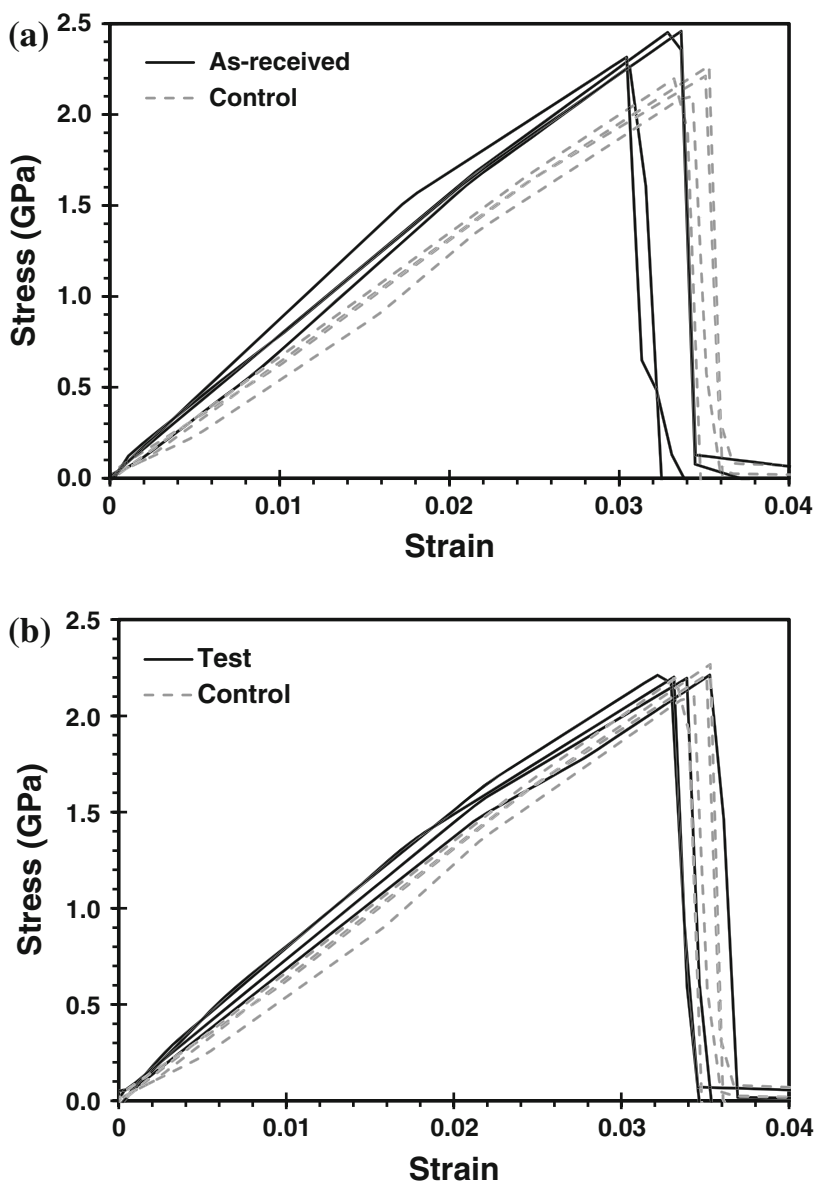

Fig. 6 Stress-strain plots from tensile tests performed on the yarn samples, a annealed (control) and non-annealed (as-received), b test and control yarns. The test yarns in (b) were evaluated at $168 \mathrm{~h}$ (i.e., 1 week) after releasing the $24 \mathrm{~h}$ creep load

Table 1 Summary of UHMWPE yarn tensile test results

\begin{tabular}{llll}
\hline & Test & Control & As-received \\
\hline Tensile strength, $\sigma_{\mathrm{f}}(\mathrm{GPa})$ & 2.21 & 2.10 & 2.27 \\
& 2.21 & 2.20 & 2.46 \\
& 2.20 & 2.21 & 2.36 \\
& 2.20 & 2.27 & 2.32 \\
Mean $\pm \mathrm{SE}$ & $2.21 \pm 0.00$ & $2.19 \pm 0.03$ & $2.35 \pm 0.04$ \\
\hline Modulus, $E(\mathrm{GPa})$ & 63.36 & 64.44 & 76.67 \\
& 68.83 & 67.03 & 72.82 \\
& 65.15 & 64.14 & 74.17 \\
Mean $\pm \mathrm{SE}$ & 65.37 & 65.71 & 78.01 \\
\hline Strain to failure, $\varepsilon_{\mathrm{f}}(\%)$ & $65.68 \pm 1.14$ & $65.33 \pm 0.66$ & $75.41 \pm 1.18$ \\
& 3.50 & 3.40 & 3.10 \\
& 3.20 & 3.30 & 3.40 \\
Mean $\pm \mathrm{SE}$ & 3.30 & 3.50 & 3.40 \\
& 3.40 & 3.50 & 3.00 \\
\end{tabular}

$S E$ standard error 

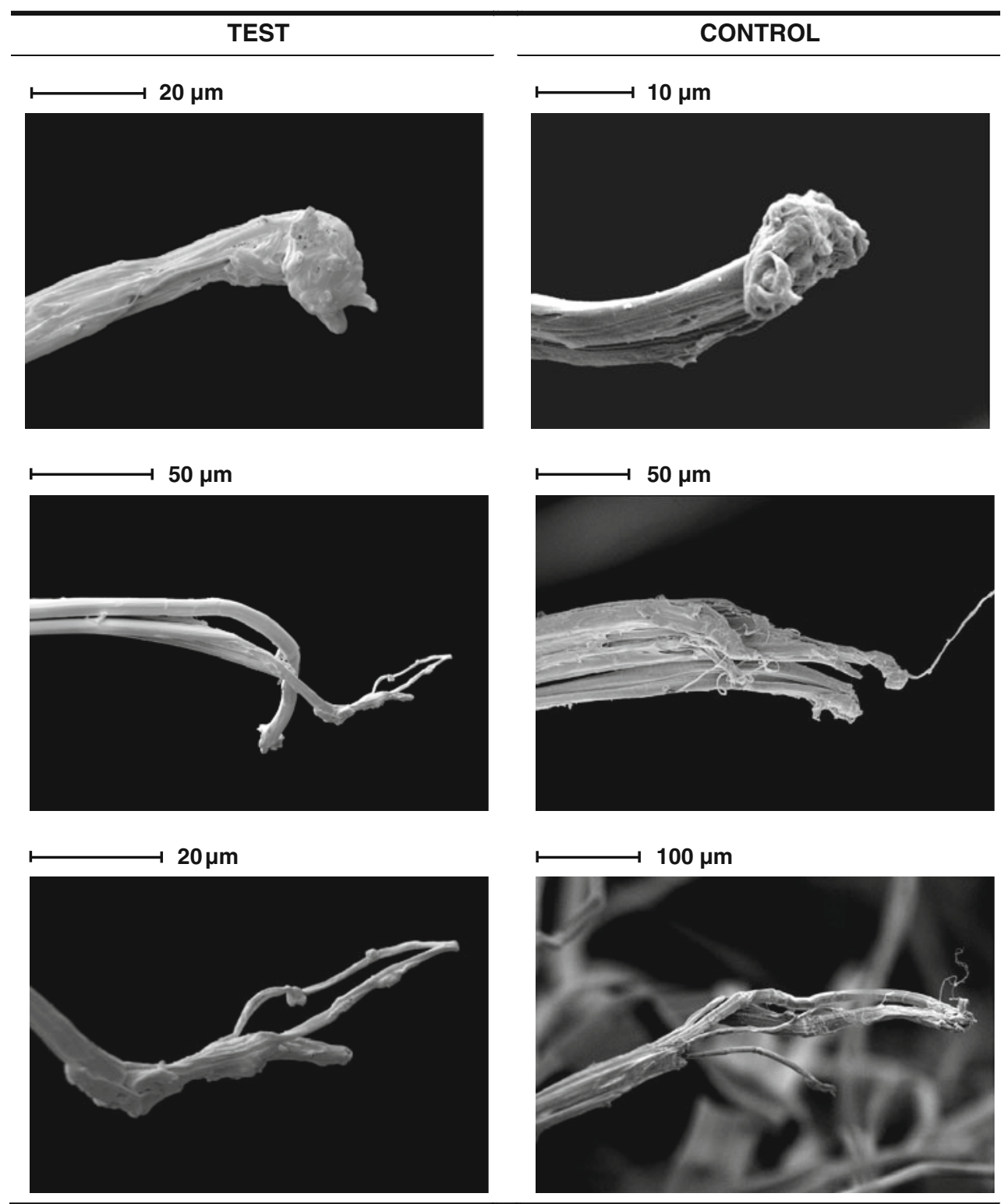

Fig. 7 Examples of fractured filament ends from test and control samples, showing similar characteristics (left-right) after tensile testing. Note changes in magnification

suggest that a secondary (competing) mechanism working against recovery force output becomes increasingly prominent.

Fitting Eq. (2) to the first 8 h of data in Fig. 8 shows that $\beta<1$, i.e., as with nylon 6,6 yarn, the force growth rate decreases with time. Although output is predicted to increase progressively towards a limiting value (12.0 $\mathrm{MPa}$ ) as $t \rightarrow \infty$ for the nylon yarn [18], Eq. (2) for the UHMWPE shows a limiting value of $21.5 \mathrm{MPa}$ beyond the first $8 \mathrm{~h}$. This may be an indication of effects from the secondary mechanism influencing the parameter values of Eq. (2) before they become visibly apparent in the output characteristic.
Analysis of composite samples

Composite sample cross-sections showing fibre spatial distributions are shown in Fig. 9. These clearly show variations in distribution, with a tendency for most fibres being in the lower half $\left(3.6 \% V_{\mathrm{f}}\right)$ or $2 / 3\left(7.2 \% V_{\mathrm{f}}\right)$ of the moulding. This effect has also been observed in open-cast nylon fibre VPPMC samples with a polyester resin matrix, as used for the previous flexural study [9] and Charpy impact testing [4-7, 10, 11]. Nevertheless, the main observation here is that for each $V_{\mathrm{f}}$ value, Fig. 9 shows no general differences in spatial distributions between test and control samples. Thus, as with the previous study [9], 
mounting all samples (in Fig. 2c) with the fibre-rich side facing downwards ensured that flexural stiffness was maximised.

Figure 10 and Table 2 summarise the bend test results and the most significant observation is that the viscoelastic prestress effect increases flexural stiffness by typically 20-40 \%. Also, as seen in Fig. 10, there appears to be no deterioration in test (or control) modulus values over the timescale investigated.

In Table 2, the control samples show average modulus values increasing with $V_{\mathrm{f}}$, from $3.4 \mathrm{GPa}\left(3.6 \% V_{\mathrm{f}}\right)$ to $4.1 \mathrm{GPa}\left(7.2 \% V_{\mathrm{f}}\right)$, i.e., the modulus is $\sim 20 \%$ higher. For the test samples, this is less, at $\sim 7 \%(4.7-5.0 \mathrm{GPa})$. There is, however, considerable variation in one of the $3.6 \% V_{\mathrm{f}}$

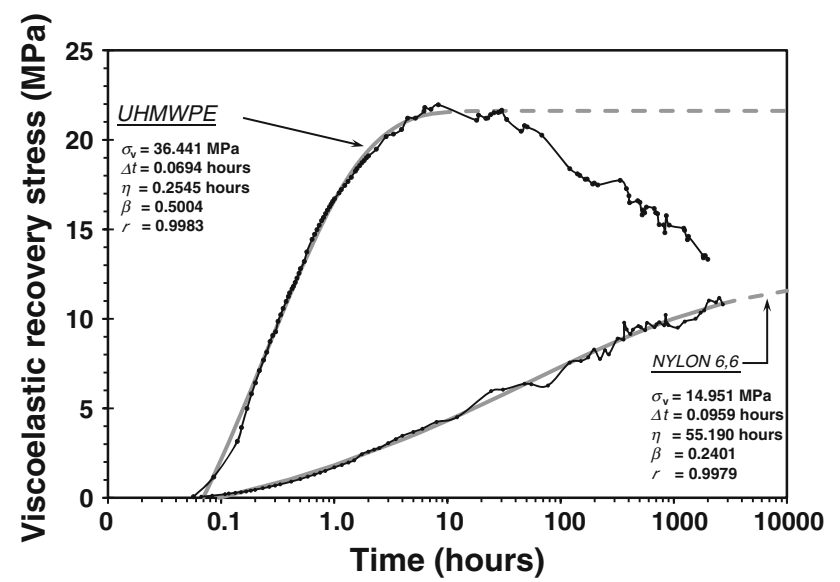

Fig. 8 Viscoelastic recovery force in terms of axial stress output (force relative to total cross-sectional area of filaments) for the UHMWPE yarn (from $1.36 \mathrm{GPa}, 24 \mathrm{~h}$ creep). For comparison, data derived from Ref. [18] is also shown for nylon 6,6 yarn (from $0.32 \mathrm{GPa}, 24 \mathrm{~h}$ creep). Equation (2) is fitted to the UHMWPE (first $8 \mathrm{~h}$ ) and nylon data, with listed parameters and correlation coefficient, $r$ batches at $336 \mathrm{~h}$ in Table 2 (giving a $145 \%$ stiffness increase between test and control samples). Excluding this batch from the data raises the average control and test sample moduli by $\sim 17$ and $\sim 13 \%$, respectively, as $V_{\mathrm{f}}$ is increased from 3.6 to $7.2 \%$; i.e., although the difference is reduced, it is not negligible. Moreover, during testing (and subsequent checking of video recordings), there appeared to be no assignable causes to such variation, hence there is no justification in excluding this batch result at $336 \mathrm{~h}$. The results thus suggest that the contribution to increased bending stiffness arising from viscoelastic prestress mechanisms may become less effective at higher $V_{\mathrm{f}}$ values.

\section{Discussion}

Viscoelastic recovery force from UHMWPE fibres

The possibility of two counteracting mechanisms causing the unexpected output characteristic for UHMWPE fibres in Fig. 8 requires further consideration, especially since recovery strain data in Fig. 4 shows no comparable trend. The stretching stage ( $\operatorname{Rig} A$ ), required the yarn to be wound (twice) around the lower bobbin to minimise stress concentration problems and this set-up was maintained as the assembly was transferred to the FM rig (Fig. 2b). Thus, at least some of the decreasing recovery in Fig. 8 could be attributed to gradual friction-affected slipping of the yarn around the lower bobbin, reducing force output from the main loop. Preliminary tests, however, had been conducted where (following the stretching stage), the yarn was refitted to the lower bobbin after removing the wound material. Although unavoidable fibre damage affected force output, a similar trend in output with time was

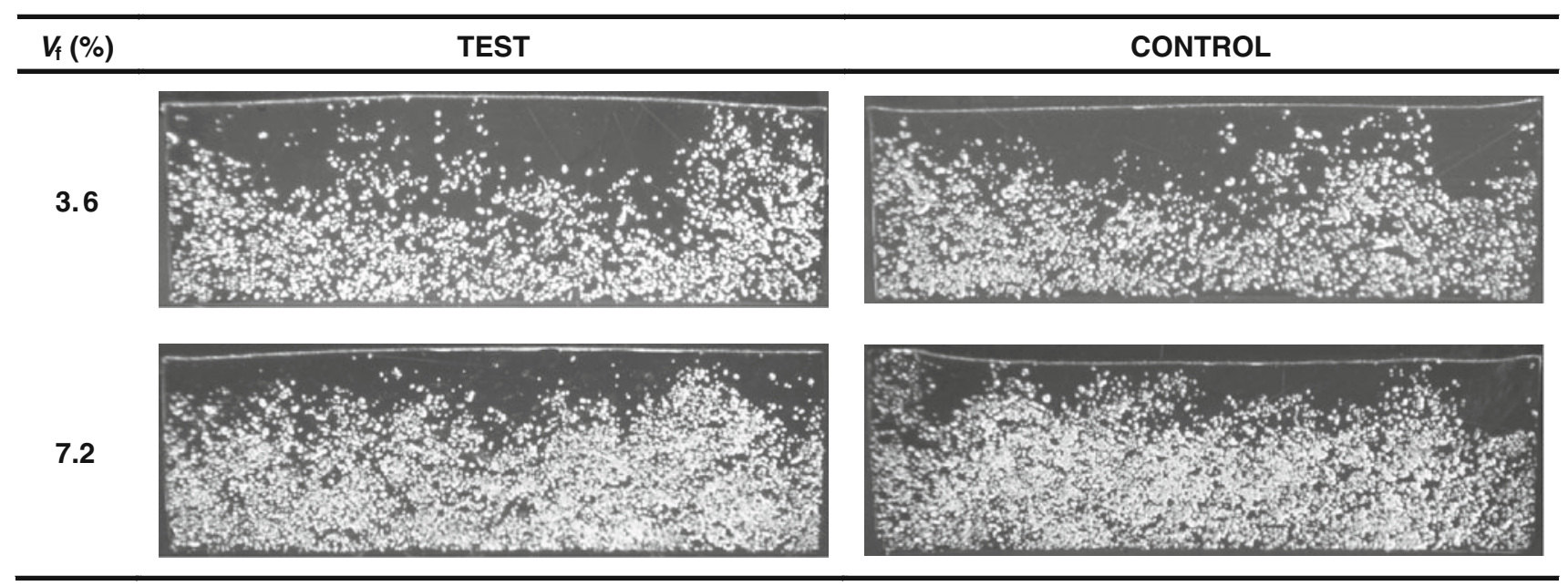

Fig. 9 Representative optical micrograph (polished) sections of all composite samples investigated 
observed, suggesting that experimentally induced yarn slippage was not the primary cause.

This leads to the conclusion that the two mechanisms are structurally based and structural differences may emanate from fibre heterogeneity. Researchers have referred to gel-spun UHMWPE fibres possessing skin-core properties, the skin most likely consisting of low molecular weight fragments and solvent excluded during crystallisation [21, $30]$, or as an unconstrained layer around a constrained core [31]. Etching experiments [26] have revealed long narrow density-deficient regions within the crystal structure of the core, resulting from contraction-induced stresses during crystallisation, an effect not occurring within the skin. Through microdiffraction experiments with a single UHMWPE fibre, Riekel et al. [32] have identified the

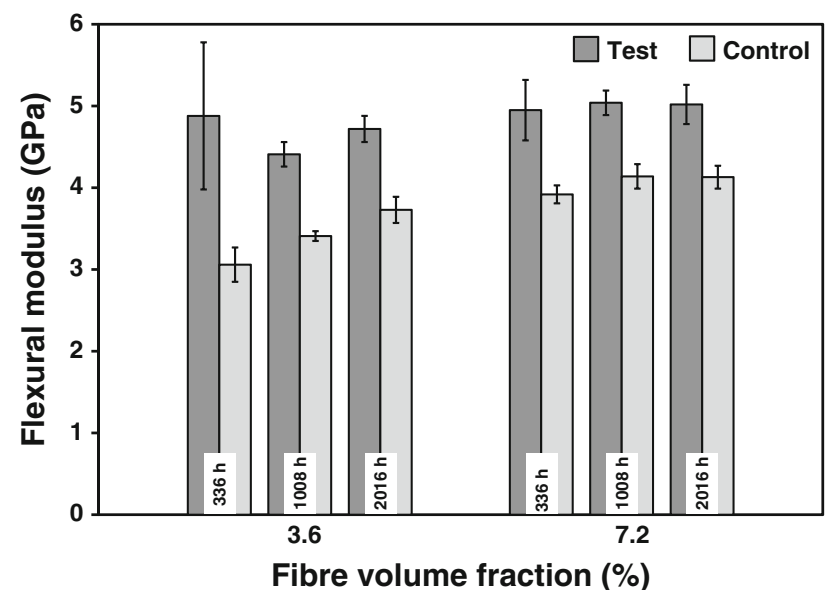

Fig. 10 Flexural modulus values determined from the three-point bend tests. Each value represents the mean of three samples with corresponding standard error possibility of a band of monoclinic phase material extending around the filament circumference, i.e., crystallographic differences between core and outer layers. Thus, although highly speculative, a variation in mechanical characteristics across each filament, in which the filament core is stiffer and time constants for viscoelastic mechanisms are shorter than for the outer skin, enables an explanation to be proposed, as follows.

Initially in Fig. 8, the recovery force climbs within the first $8 \mathrm{~h}$ due to the filament core regions causing a rapid build-up of force as they attempt viscoelastic retraction (at fixed strain). The rate of force build-up progressively decreases as energy storage sites within the cores become depleted through force generation and possibly energy transfer to skin regions. At $\sim 8 \mathrm{~h}$, longer-term viscoelastic activity from the skin regions starts to become dominant. At this point, the force magnitude cannot be maintained by the (less stiff) skin regions, thus recovery force decreases. Ultimately, we believe this decrease will lead to an output level that should result in a state of equilibrium existing between skin and core regions. Thus eventually, the UHMWPE plot in Fig. 8 is expected to approach a constant (non-zero) value.

Since recovery strain (Fig. 4) is derived from free movement of fibres and is determined by measurements confined to the skin regions, the resulting data are insensitive to the competing effects from these core-skin interactions. Similarly, the increased bending stiffness in VPPMC samples (Fig. 10, Table 2) depends on shear stress transfer between fibre skin regions and surrounding matrix, in contrast with the axial stress shown in Fig. 8. Although no deterioration in prestress effects within the VPPMC samples is observed over the timescale investigated, Fig. 8 shows a drop in output of $\sim 25 \%$ over the same time

Table 2 Flexural modulus data from the three-point bend tests

\begin{tabular}{|c|c|c|c|c|c|c|}
\hline \multirow{3}{*}{ Age (hours) } & \multicolumn{3}{|l|}{$3.6 \% V_{\mathrm{f}}$} & \multicolumn{3}{|l|}{$7.2 \% V_{\mathrm{f}}$} \\
\hline & \multicolumn{2}{|c|}{ Flexural modulus (GPa) } & \multirow[t]{2}{*}{ Increase $(\%)$} & \multicolumn{2}{|c|}{ Flexural modulus (GPa) } & \multirow[t]{2}{*}{ Increase $(\%)$} \\
\hline & Test & Control & & Test & Control & \\
\hline \multirow[t]{3}{*}{336} & 3.74 & 3.03 & 23.69 & 5.65 & 4.05 & 39.52 \\
\hline & 4.24 & 3.44 & 23.15 & 4.81 & 4.01 & 19.92 \\
\hline & 6.65 & 2.71 & 145.23 & 4.38 & 3.70 & 18.24 \\
\hline \multirow[t]{3}{*}{1008} & 4.28 & 3.49 & 22.52 & 4.99 & 3.85 & 29.58 \\
\hline & 4.71 & 3.44 & 36.83 & 4.81 & 4.25 & 13.25 \\
\hline & 4.23 & 3.30 & 28.52 & 5.31 & 4.32 & 23.07 \\
\hline \multirow[t]{3}{*}{2016} & 4.73 & 3.95 & 19.79 & 5.30 & 4.28 & 23.91 \\
\hline & 4.99 & 3.81 & 31.08 & 4.81 & 4.01 & 19.92 \\
\hline & 4.44 & 3.42 & 29.83 & 4.96 & 4.09 & 21.24 \\
\hline Mean \pm SE & $4.67 \pm 0.28$ & $3.40 \pm 0.12$ & $40.07 \pm 13.26$ & $5.00 \pm 0.12$ & $4.06 \pm 0.07$ & $23.18 \pm 2.52$ \\
\hline
\end{tabular}

$S E$ standard error 
period (336-2016 h). This lends support to the view that the skin regions have the dominant role in longer term viscoelastic activity.

In terms of force output characteristics, other aspects may require further consideration, e.g., the effects of (i) annealing and (ii) filament geometry and substructure. For (i), despite only small changes in short-term mechanical properties (Fig. 6a; Table 1), the annealing treatment has a major effect on viscoelastic activity, as demonstrated in Figs. 3 and 4. Thus recovery force must also be affected and perhaps, skin-core effects. X-ray diffraction results for Dyneema at $120^{\circ} \mathrm{C}$ [31] (our annealing temperature) indicate some crystalline rearrangement may have occurred, and strain relaxation within the amorphous regions can also be expected [14, 33] but the optimum annealing conditions for recovery force output would require further investigation. For (ii), as indicated by Figs. 5 and 7, the filament cross-sections are not circular; also they have a substructure of typically 150 macrofibrils, a macrofibril being $0.5-2 \mu \mathrm{m}$ in diameter [23]. Thus filaments and their macrofibrils have variations in section area; hence for smaller section areas, skin-related effects may be expected to be more significant.

\section{Influence of prestress mechanisms on flexural modulus}

Various mechanisms have been speculated to explain how prestress may increase flexural modulus [9] but the current findings may facilitate further understanding. As reported earlier, the results in Fig. 10 and Table 2 suggest that the contribution to flexural stiffness from prestress does not increase as fast as the actual fibre contribution when $V_{\mathrm{f}}$ is increased from 3.6 to $7.2 \%$. Thus although flexural modulus is $\sim 40 \%$ higher at $3.6 \% V_{\mathrm{f}}$, this drops to $\sim 23 \%$ at $7.2 \% V_{\mathrm{f}}$. Reasons for this reduction as $V_{\mathrm{f}}$ increases could include effects of (i) deflection-dependent forces, (ii) an optimum $V_{\mathrm{f}}$ value, and (iii) changes in fibre spatial distribution.

For (i), flexural modulus may be increased by a mechanism proposed for elastically prestressed (glass fibreepoxy resin) composites [3]. Here, the applied (downwards) bending force is opposed by residual tension in the fibres which creates a vertical (upwards) force component, the latter increasing as bending angle (deflection) increases. Thus, there will be less deflection in bending (at a given load) for a stiffer material, i.e., as $V_{\mathrm{f}}$ is increased: hence this prestress-induced stiffening mechanism would be expected to become less effective.

For (ii), tensile testing of VPPMC samples [8] showed that maximum improvements in mechanical properties occurred at $\sim 35-40 \% V_{\mathrm{f}}$. This was attributed to competing effects of fibres: too few fibres create less compressive stress within the matrix, whereas too many fibres reduce the cross-sectional area over which compressive stresses can operate. We suggest that an optimum $V_{\mathrm{f}}$ may also occur in flexure, but the mechanisms influencing its value will be more complex than the situation observed with VPPMCs in simple tension. For example, external loading imposes a combination of tensile and compressive stresses in bending thus an optimum $V_{\mathrm{f}}$ value may depend on flexural deflection conditions. Also, the effects of nonuniform fibre spatial distribution (Fig. 9) will influence $I$ in Eq. (3): if most fibres lie close to the lower surface (subjected to tension during bending), the optimum (whole sample) $V_{\mathrm{f}}$ value for maximising bending stiffness from prestress may be significantly lower than the case for axially applied tensile loads.

For (iii), in addition to the effects of non-uniform fibre spatial distribution on (ii), any changes in this distribution over composite cross-sectional area as $V_{\mathrm{f}}$ is increased will also affect prestress contributions. As stated earlier, Fig. 9 shows the fibres at $3.6 \% V_{\mathrm{f}}$ being mainly confined to the lower half of the sample, but this increases to $2 / 3$ at $7.2 \%$ $V_{\mathrm{f}}$. Thus effectiveness of the prestress contribution to bending stiffness is reduced in the latter case, since fibre distribution extends further from the lower surface. To some extent, (iii) may also be supported by the observation that previous three-point bend tests on composites using nylon fibre in epoxy resin at 8.0-16\% $V_{\mathrm{f}}$ [9] had relatively uniform fibre spatial distributions and these showed no significant prestress-related dependency on $V_{\mathrm{f}}$.

\section{Conclusions}

This work reports on initial investigations into the potential of UHMWPE fibres for providing viscoelastically generated prestress within a composite material. The main findings are:

(i) By using appropriate annealing and creep conditions, long-term viscoelastic recovery strain can be achieved, which suggests that these fibres can release mechanical energy over a long timescale.

(ii) The selected annealing conditions have only a minor effect on the short-term (tensile) mechanical properties of these fibres.

(iii) Annealed fibres, after being subjected to the selected creep parameters, show no significant differences in short-term mechanical properties or fibre topography.

(iv) A viscoelastically generated recovery force is demonstrated; however, the force output-time characteristic indicates that two competing mechanisms could be occurring. We suggest this may arise from skincore interactions occurring within the fibres, caused 
by differences in viscoelastic properties between skin and core regions.

(v) The viability of VPPMCs using UHMWPE fibres is demonstrated through three-point bend tests. Compared with control (unstressed) counterparts, these VPPMC samples show mean increases in flexural stiffness of 40 and $23 \%$ at 3.6 and $7.2 \% V_{\mathrm{f}}$, respectively, with no deterioration in modulus values over the timescale $(\sim 2000 \mathrm{~h})$ investigated. The lower increase at $7.2 \% V_{\mathrm{f}}$ may arise from effects relating to deflection-dependent forces, optimum fibre-matrix ratio and changes in fibre spatial distribution within the composite as $V_{\mathrm{f}}$ is increased.

Some of the findings raise issues concerning the fundamental properties of UHMWPE fibres and our proposed explanations are speculative. Nevertheless, this work also provides sufficient evidence to demonstrate that these fibres should have an important role in the future development of VPPMC technology.

Acknowledgements The authors would like to thank Garry Robinson for technical support. Departmental support $(\mathrm{PhD}$ degree fee waiver) for one of the authors (AF) is gratefully acknowledged.

\section{References}

1. Hadi AS, Ashton JN (1998) Compos Struct 40:305

2. Motahhari S, Cameron J (1998) J Reinf Plast Compos 17:123

3. Motahhari S, Cameron J (1999) J Reinf Plast Compos 18:279

4. Fancey KS (2005) J Adv Mater 37:21

5. Fancey KS (2000) Mater Sci Eng A 279:36

6. Fancey KS (2000) J Reinf Plast Compos 19:1251

7. Pang JWC, Fancey KS (2006) Mater Sci Eng A 431:100
8. Pang JWC, Fancey KS (2008) Compos Sci Technol 68:1903

9. Pang JWC, Fancey KS (2009) Compos Part A 40:784

10. Fancey KS (2010) Compos Part B 41:454

11. Fazal A, Fancey KS (2013) Compos Part B 44:472

12. Gupta VB (2002) J Appl Polym Sci 83:586

13. Devaux E, Caze C (1999) Compos Sci Technol 59:459

14. Hu W, Buzin A, Lin JS, Wunderlich B (2003) J Polym Sci Part B Polym Phys 41:403

15. Fancey KS (2001) J Polym Eng 21:489

16. Fancey KS (2005) J Mater Sci 40:4827. doi:10.1007/s10853-0052020-x

17. Lamin BM (2005) B.Eng. Thesis, Dept Engineering, University of Hull, UK

18. Pang JWC, Lamin BM, Fancey KS (2008) Mater Lett 62:1693

19. Timoshenko S (1955) Strength of materials, Part I. Elementary theory and problems, 3rd edn. Krieger, New York (1976 reprint)

20. Tan VBC, Zeng XS, Shim VPW (2008) Int J Impact Eng 35:1303

21. Silverstein MS, Breuer O (1993) J Mater Sci 28:4153. doi:10.1007/BF00351246

22. Grubb DT, Li ZF (1994) J Mater Sci 29:203. doi:10.1007/ BF00356593

23. Berger L, Kausch HH, Plummer CJG (2003) Polymer 44:5877

24. Peijs T, Smets EAM, Govaert LE (1994) Appl Compos Mater $1: 35$

25. Van der Werff H, Pennings AJ (1991) Colloid Polym Sci 269:747

26. Abo El-Maty MI, Olley RH, Bassett DC (1999) J Mater Sci 34:1975. doi:10.1023/A:1004547101567

27. Dijkatra DJ, Pennings AJ (1988) Polym Bull 19:481

28. Smook J, Hamersma W, Pennings AJ (1984) J Mater Sci 19:1359. doi:10.1007/BF01120049

29. Languerand DL, Zhang H, Murthy NS, Ramesh KT, Sansoz F (2009) Mater Sci Eng A 500:216

30. Tissington B, Pollard G, Ward IM (1991) J Mater Sci 26:82. doi:10.1007/BF00576036

31. Kakiage M, Tamura T, Murakami S, Takahashi H, Yamanobe T, Uehara H (2010) J Mater Sci 45:2574. doi:10.1007/s10853-0104228-7

32. Riekel C, Cedola A, Heidelbach F, Wagner K (1997) Macromolecules 30:1033

33. Peterlin A (1977) J Appl Phys 48:4099 